\title{
MED12 regulates a transcriptional network of calcium-handling genes in the heart
}

\author{
Kedryn K. Baskin, ${ }^{1}$ Catherine A. Makarewich, ${ }^{1}$ Susan M. DeLeon, ${ }^{1}$ Wenduo Ye, ${ }^{1}$ Beibei Chen, ${ }^{2}$ \\ Nadine Beetz, ${ }^{1}$ Heinrich Schrewe, ${ }^{3}$ Rhonda Bassel-Duby, ${ }^{1,4,5}$ and Eric N. Olson ${ }^{1,4,5}$ \\ 'Department of Molecular Biology and ${ }^{2}$ Department of Clinical Sciences, University of Texas Southwestern Medical Center, \\ Dallas, Texas, USA. ${ }^{3}$ Department of Developmental Genetics, Max Planck Institute for Molecular Genetics, Berlin, Germany. \\ ${ }^{4}$ Hamon Center for Regenerative Science and Medicine and ${ }^{5}$ Sen. Paul D. Wellstone Muscular Dystrophy Cooperative \\ Research Center, University of Texas Southwestern Medical Center, Dallas, Texas, USA.
}

\begin{abstract}
The Mediator complex regulates gene transcription by linking basal transcriptional machinery with DNA-bound transcription factors. The activity of the Mediator complex is mainly controlled by a kinase submodule that is composed of 4 proteins, including MED12. Although ubiquitously expressed, Mediator subunits can differentially regulate gene expression in a tissue-specific manner. Here, we report that MED12 is required for normal cardiac function, such that mice with conditional cardiac-specific deletion of MED12 display progressive dilated cardiomyopathy. Loss of MED12 perturbs expression of calcium-handling genes in the heart, consequently altering calcium cycling in cardiomyocytes and disrupting cardiac electrical activity. We identified transcription factors that regulate expression of calcium-handling genes that are downregulated in the heart in the absence of MED12, and we found that MED12 localizes to transcription factor consensus sequences within calcium-handling genes. We showed that MED12 interacts with one such transcription factor, MEF2, in cardiomyocytes and that MED12 and MEF2 co-occupy promoters of calcium-handling genes. Furthermore, we demonstrated that MED12 enhances MEF2 transcriptional activity and that overexpression of both increases expression of calcium-handling genes in cardiomyocytes. Our data support a role for MED12 as a coordinator of transcription through MEF2 and other transcription factors. We conclude that MED12 is a regulator of a network of calciumhandling genes, consequently mediating contractility in the mammalian heart.
\end{abstract}

Authorship note: W. Ye is deceased.

Conflict of interest: The authors have declared that no conflict of interest exists.

Submitted: November 22, 2016

Accepted: June 13, 2017

Published: July 20, 2017

\section{Reference information:}

JCI Insight. 2017;2(14):e91920.

https://doi.org/10.1172/jici.

insight.91920.

\section{Introduction}

Heart failure is the leading cause of death in the Western world (1). Defective calcium $\left(\mathrm{Ca}^{2+}\right)$ cycling, such as decreased $\mathrm{Ca}^{2+}$ uptake in the sarcoplasmic reticulum (SR) or SR calcium leak occurs in failing hearts $(2,3)$, and genetic mutations in calcium-handling genes cause arrhythmias and dilated cardiomyopathy (DCM) (4). Altered expression of calcium-handling genes perturbs contractility (5-9), but relatively little is known about how the expression of these genes is coordinated in the heart.

Mediator is a multiprotein complex of about 30 subunits that form the core and kinase submodules (10, 11). The kinase submodule, containing MED12, MED13, CDK8, and Cyclin C (12), can repress transcription through allosteric inhibition of RNA Pol II binding to the core submodule (13) but can also activate transcription by promoting Pol II recruitment to target genes via specific transcription factors (14). We previously demonstrated that cardiac MED13 regulates systemic energy homeostasis through signaling to extracardiac tissues $(15,16)$, but the roles of the other kinase components in the heart have not been investigated.

MED12, a component of the Mediator kinase submodule, is encoded on the $\mathrm{X}$ chromosome, and missense mutations are associated with a variety of X-linked disorders (17-19). Developmental signaling pathways and transcription factors converge on MED12, which acts as a transcriptional hub required to coordinate development (20-23). Deletion of Med12 in Drosophila decreases expression of sonic hedgehog target genes and leads to defects in eye development (20), and mutations in Med12 in zebrafish disrupt SOX-mediated transcription during endoderm development (22). MED12 is also required for neuron development through its regulation of TBX2B in zebrafish (23). Med12 hypomorphic mutant mice die in utero due to various developmental defects $(24,25)$, but the function of MED12 in the heart has not yet been investigated. 
In this study, we investigated the function of MED12 in the heart by deleting Med12 in cardiomyocytes (CMs). Mice with cardiac-specific deletion of Med12) suffer from early-onset DCM. Loss of Med12 alters expression of calcium-handling genes, ultimately disrupting calcium cycling in CMs and resulting in decreased cardiac function. MED12 regulates expression of calcium-handling genes in part by coordinating transcription through MEF2 and several other transcription factors, but loss of MED12 does not affect MEF2-DNA binding or RNA Pol II recruitment. Our findings demonstrate that MED12 controls the transcriptional network of calcium-handling genes, consequently mediating contractility in the heart.

\section{Results}

Cardiac-specific deletion of Med12 causes DCM. Med12 is highly expressed in the heart during early embryonic development but declines after E15.5 and is expressed at similar levels in the neonatal and adult mouse heart (Figure 1A). To investigate the function of MED12 in the heart, we crossed Med12 $2^{\text {flfl}}$ female mice (24) with male mice expressing the $\alpha M h c$-Cre transgene, which is expressed in a CM-specific manner (26). $\alpha M h c-C r e^{+}$male mice with a single-floxed $\mathrm{Med} 12$ allele $\left(\alpha \mathrm{Mhc}-\mathrm{Cre}^{+} \mathrm{Med} 12^{f / y}\right.$, abbreviated as Med12cKO) had significantly less cardiac Med12 expression than $\alpha M h c-C r e-M e d 12^{f / y}$ (CTL) mice in whole heart (Figure 1B). Because $\alpha M h c$-Cre expression is CM specific, Med12 mRNA was not detected in CMs isolated from Med$12 c K O$ hearts (Figure 1B), confirming efficient deletion of Med12.

Male and female $\mathrm{Med} 12 \mathrm{cKO}$ mice were born at the expected Mendelian ratios and appeared identical to littermate controls. Hearts of $\mathrm{Med} 12 \mathrm{cKO}$ mice were indistinguishable morphologically from CTL hearts at birth but displayed ventricular wall thinning and dilation by postnatal day 7 (P7) (Figure 1C). By 5 weeks of age, Med12cKO hearts weighed significantly more than CTL hearts, which was exacerbated at 12 weeks of age (Figure 1D). Echocardiography performed on unanesthetized male mice revealed parameters indicative of cardiac dysfunction. Fractional shortening, a measure of cardiac contractility, was significantly reduced in Med12cKO mice as early as P7 and declined progressively with age (Figure 1E and Supplemental Figure 1; supplemental material available online with this article; https://doi.org/10.1172/ jci.insight.91920DS1). Heart rate was significantly decreased in Med12cKO mice by P7 and remained lower throughout adulthood (Figure 1F). Left ventricular posterior wall thickness in diastole (LVPWD) and systole (LVPWS) were decreased by P7 and continued to decline, indicating early and progressive thinning of the left ventricular wall (Figure 1, G and H). Left ventricular internal dimension in diastole (LVIDD) and systole (LVIDS) were already increased at P7 and continued to increase with aging, indicating significant dilation of the left ventricular chamber (Figure 1, I and J). Female mice with mosaic Med12 expression in the heart $\left(\alpha \mathrm{Mhc}-\mathrm{Cr} e^{+} \mathrm{Med} 12^{f /+}\right)$ had slightly decreased cardiac function, and complete loss of Med12 $\left(\alpha M h c-C r e^{+} \mathrm{Med} 12^{f / f f}\right)$ in females significantly decreased cardiac function to nearly the same degree as in male Med12cKO mice (Supplemental Figure 1, B and C, and Supplemental Table 1).

DCM is characterized by ventricular chamber enlargement and cardiac dysfunction with no heart wall hypertrophy. In some cases, prominent fibrosis accompanies late-stage DCM (27). Masson's trichrome staining of adult male $\mathrm{Med} 12 \mathrm{cKO}$ hearts demonstrated an increase in interstitial cardiac fibrosis (Figure 2A). Quantification of cardiac fibrosis using Picrosirius red staining revealed a slight increase in fibrosis at P7, which was significant at 12 weeks of age (Figure 2, B and C). Despite increased fibrosis in $\mathrm{Med} 12 \mathrm{CKO}$ hearts, no differences in CM death were detected (Supplemental Figure 2, A-C). Additionally, differences in CM proliferation were unchanged in hearts from $\mathrm{Med} 12 \mathrm{CKO}$ mice (Supplemental Figure 2, $\mathrm{D}-\mathrm{F})$. We also analyzed CM ultrastructure by electron microscopy and observed that sarcomeric structure in $\mathrm{Med} 12 \mathrm{cKO}$ hearts was identical to control hearts throughout most regions of the heart (Figure $2 \mathrm{D}$, middle panel), with only a few regions containing disorganized sarcomeres (Figure 2D, right panel).

To determine whether cardiac conduction is altered in Med12cKO mice, as often occurs in patients with DCM (28), electrocardiography was performed on anesthetized Med12cKO mice. Adult male Med12cKO mice had decreased heart rates when anesthetized (Figure 2E), to the same degree as observed in the unanesthetized state (Figure 1F). Loss of Med12 in the heart leads to abnormal cardiac electrical activity, specifically a prolongation of the QRS interval (Figure 2, F and G). These results indicate that Med12 is required to maintain normal cardiac function and that loss of cardiac Med12 causes early-onset DCM.

Deletion of Med12 alters expression of calcium-handling genes in the heart. To gain an understanding of how loss of Med12 leads to DCM, we performed RNA-Seq on ventricles from mice at P1, before Med12cKO mice develop DCM. We identified 103 genes that were significantly upregulated and 97 genes that were 
A

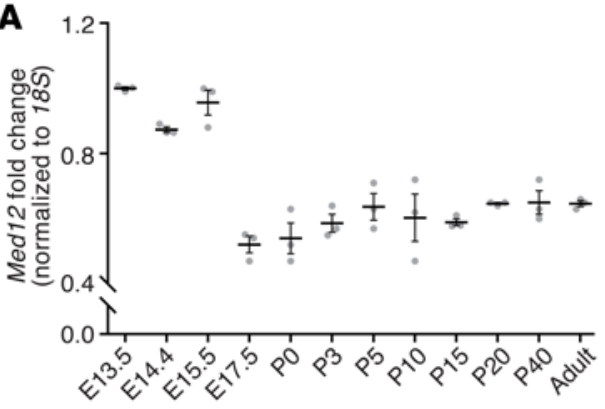

C

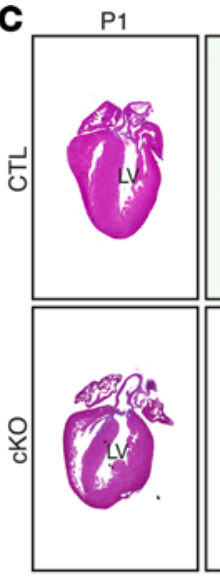

D 20
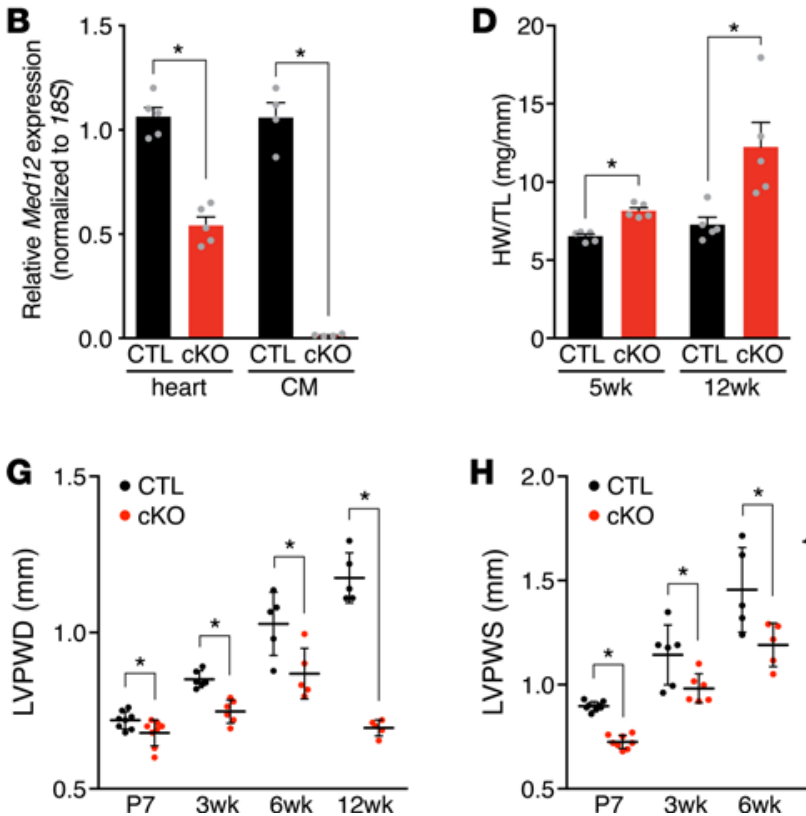
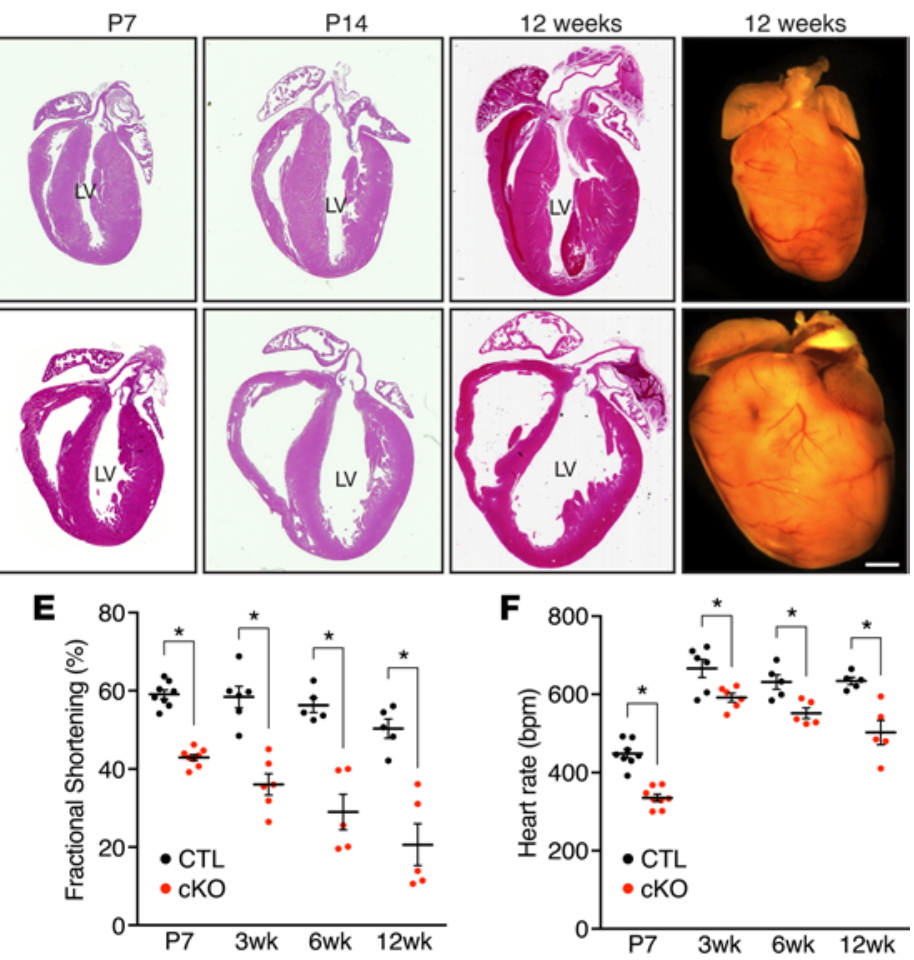

Figure 1. Deletion of Med12 in cardiomyocytes impairs cardiac function. (A) Med12 mRNA expression in ventricles during development and aging of mouse hearts. $n=3$. (B) Med12 expression in hearts $(n=5)$ and cardiomyocytes (CMs) from control (CTL) and Med12cKO (cKO) male mice $(n=4-5$ mice). (C) H\&E staining and whole-mount heart representations of CTL and cKO male hearts. Scale bars: $1 \mathrm{~mm}$. Serial sections are shown in C and Figure 2, A and B. LV, left ventricle. (D) Heart weight (HW) to tibia length (TL) analysis. $n=5$. (E) Fractional shortening, (F) heart rate, (C) left ventricular posterior wall thickness in diastole (LVPWD) and (H) in systole (LVPWS), (I) left ventricular internal dimension in diastole (LVIDD) and (J) in systole (LVIDS) of CTL and cKO male hearts. $n=5-8$. Data are mean \pm SEM. ${ }^{*} P<0.05$ by 2 -tailed Student's $t$ test.

significantly downregulated in $\mathrm{Med} 12 \mathrm{cKO}$ ventricles by at least 1.5 -fold with $P<0.05$ (Figure 3A). The Mediator kinase submodule can be composed of MED12, MED13, and CDK8 (12) or their respective paralogs MED12-like (MED12L), MED13-like (MED13L), and CDK8-like (CDK19), in a mutually exclusive manner (11). Although Med12 and Med12l have similar developmental expression patterns in the mouse heart (Figure 1A and Supplemental Figure 3A), deletion of Med12 did not lead to a compensatory upregulation of Med12l (Supplemental Figure 3B). Furthermore, expression of other Mediator subunits in the heart was unaffected by deletion of Med12 (Supplemental Figure 3B).

Gene Ontology (GO) analysis identified the top processes regulated in Med12cKO hearts as the muscle system, suggesting the importance of Med12 in regulating contractility of the heart (GSE100089; https://www.ncbi.nlm.nih.gov/geo/query/acc.cgi?acc=GSE100089) (Figure 3B). Using Ingenuity Pathway Analysis, we discovered that the top pathway altered in Med12cKO hearts was calcium signaling (Figure 3C). Based on this finding, we identified calcium-handling genes that were significantly downregulated (Atp2a2, Gja1 and -5, Kcnn1, Pln, Ryr2, and Tnnt1), and upregulated (Cacna1d, Casq1, Gja3, and Slc8a2) in Med12cKO hearts by RNA-Seq (Figure 3D). We quantified the expression of many 
A


P1
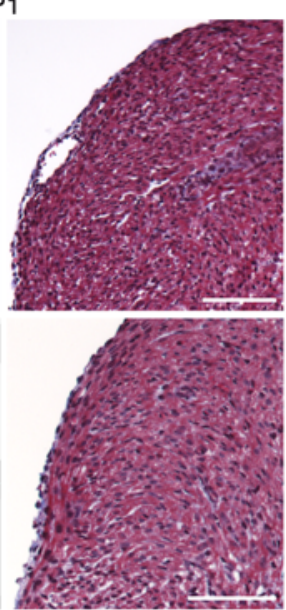

P7

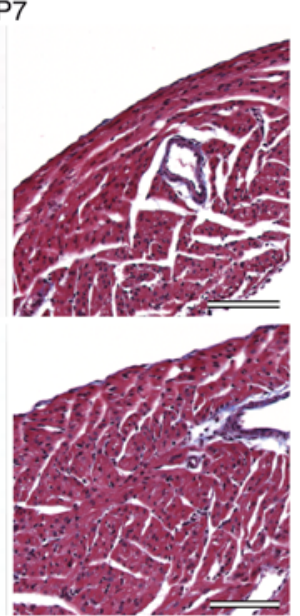



12 wks
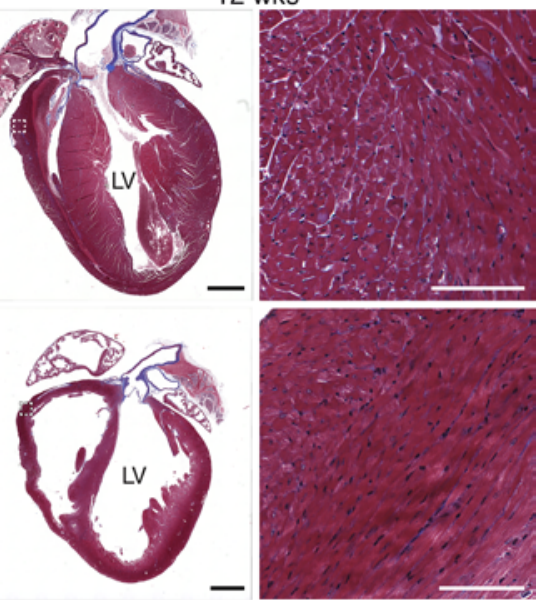

B $\quad P$

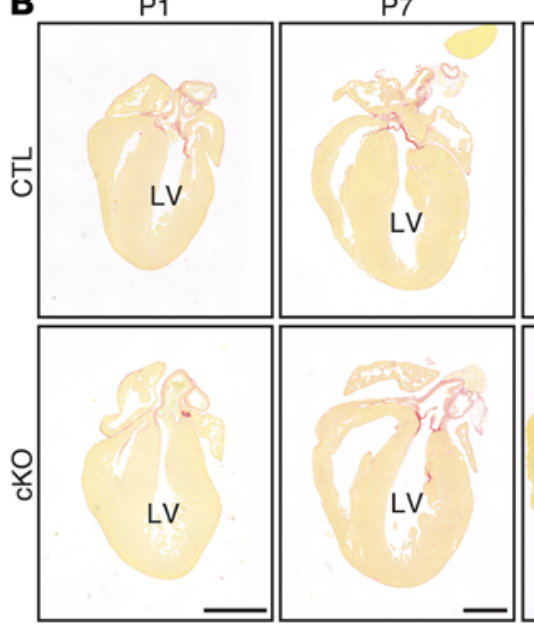

C

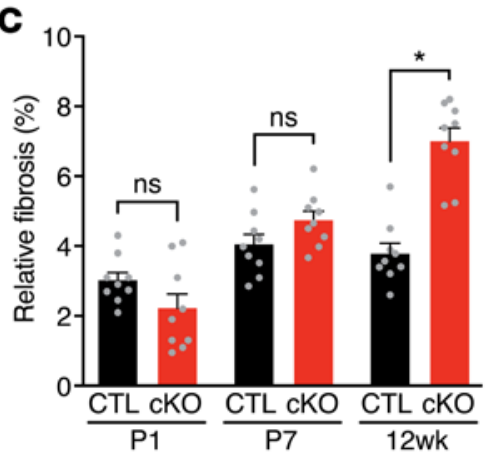

D
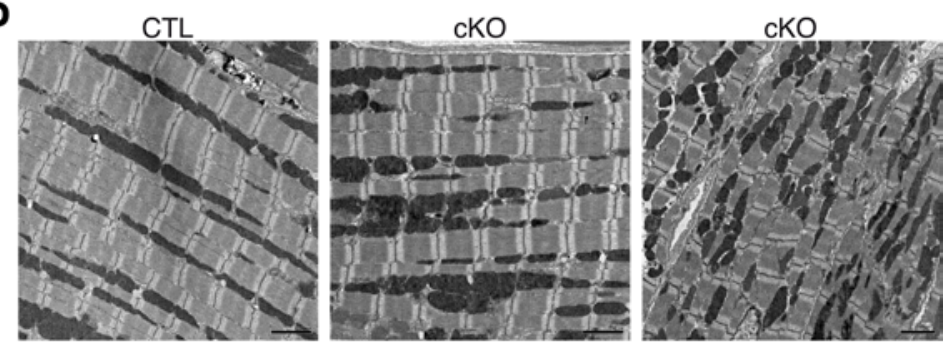

$\mathbf{F}$

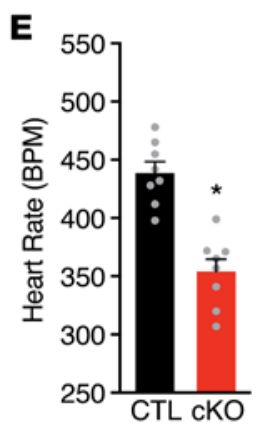

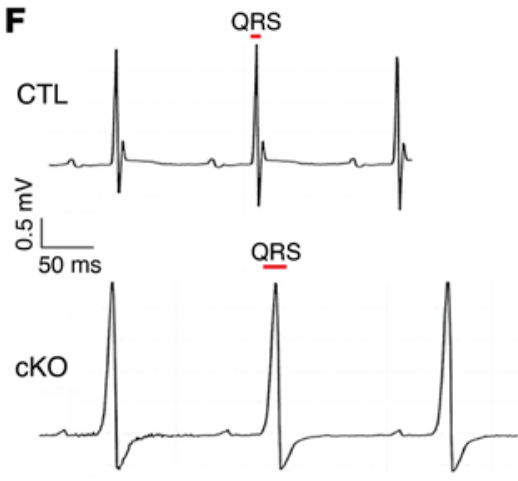

G



Figure 2. Absence of Med12 in cardiomyocytes alters electrical activity of the heart. (A) Masson's trichrome staining and (B) Picrosirius red staining of CTL and cKO hearts. Scale bars: $1 \mathrm{~mm}$. LV, left ventricle. (C) Quantification of Picrosirius red staining of fibrosis $(n=3)$ in heart sections from 3 mice per group. (D) Representative transmission electron microscopy images of CTL and cKO LVs from 8-week-old male mice. Scale bars: $2 \mu \mathrm{m}$. (E) Heart rate, (F) representative electrocardiogram tracings, and (G) quantification of QRS length in 6-week-old adult male hearts. $n=8$, E and $\mathbf{G}$. Data are mean \pm SEM. ${ }^{*} P<0.05$ by 2 -tailed Student's $t$ test.

calcium-handling genes by qPCR in independent ventricular samples from P1, P 7, P14, and 8 weekold $\mathrm{Med} 12 \mathrm{cKO}$ mice and in CMs isolated from adult hearts. All calcium-related genes investigated were significantly regulated at $\mathrm{P} 1$, before the onset of DCM, and remained significantly down- or upregulated in hearts and CMs of adult mice (Figure 3, E and F). These results suggest that gene expression perturbation precedes structural and functional changes in Med12cKO hearts.

To determine the functional consequences of altered gene expression, we quantified levels of key calcium-handling proteins. In response to an action potential, $\mathrm{Ca}^{2+}$ influx into $\mathrm{CM}$ s triggers calcium-induced 
A

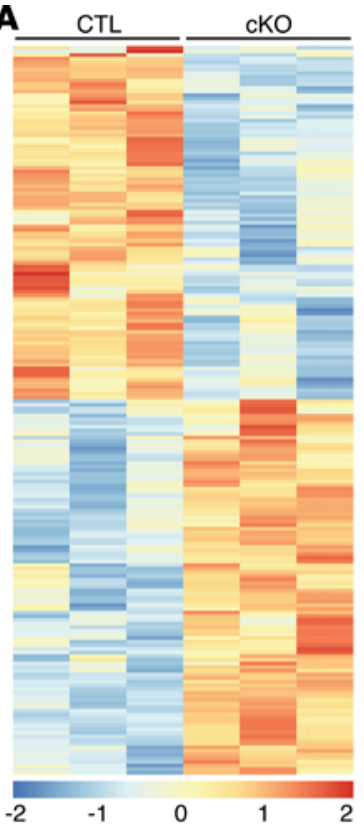

B

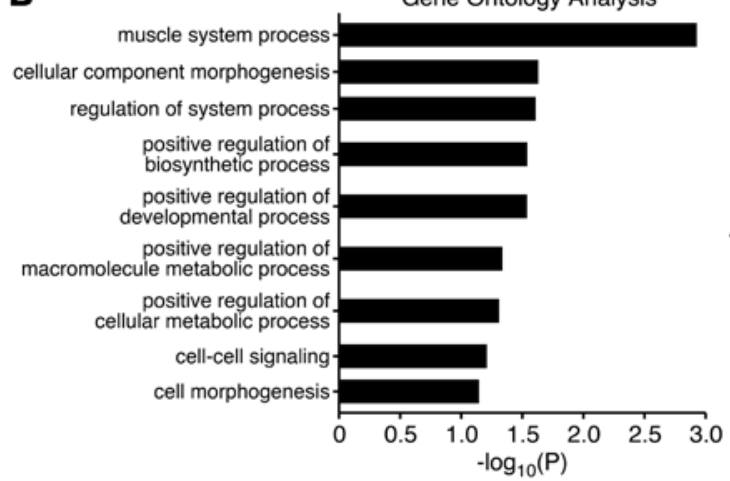

C

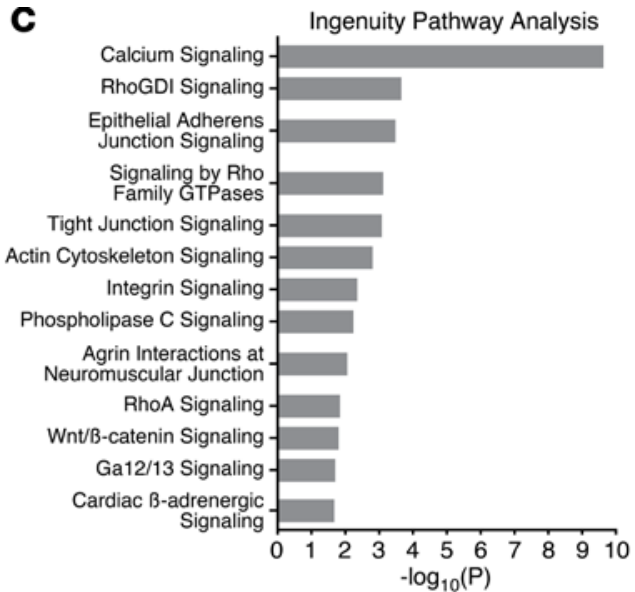

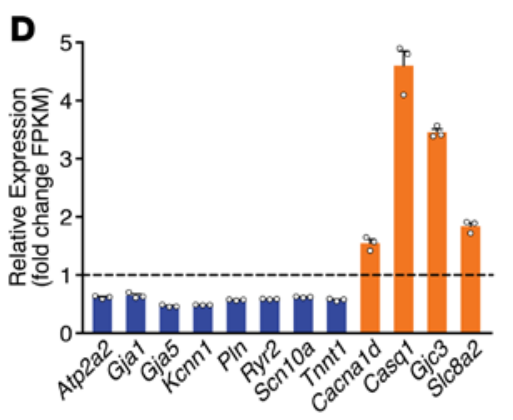

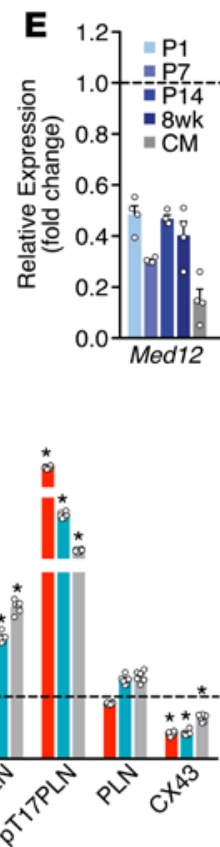

H

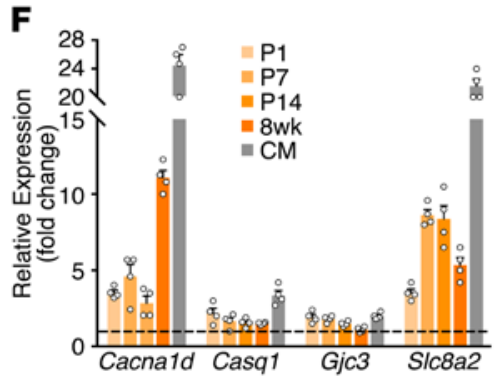

G

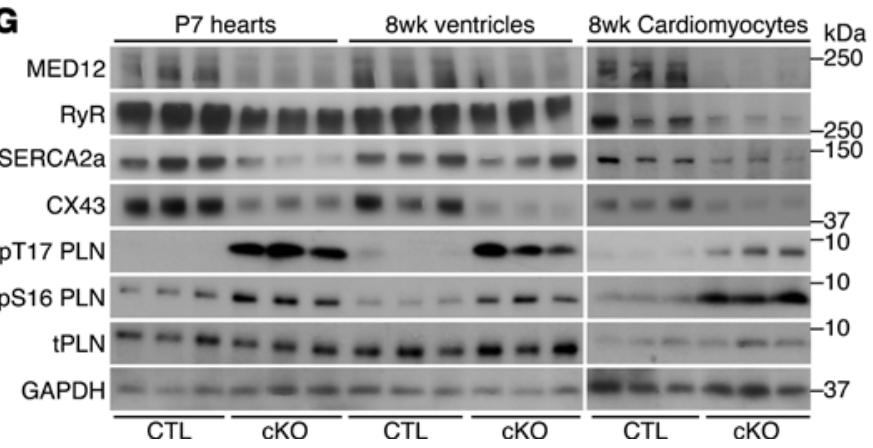

Figure 3. MED12 regulates calcium-handling genes in the heart. (A) Heat map of differentially expressed genes in CTL and Med12cKO (cKO) ventricles at $\mathrm{P} 1$, analyzed by RNA-Seq with cutoff of $P<0.05$ and 1.5 fold change. $n=3$. (B) Gene Ontology Analysis and (C) Ingenuity Pathway Analysis of differentially expressed genes in CTL and CKO ventricles. (D) Calcium-handling genes are differentially expressed in CTL and cKO ventricles (RNA-Seq data). (E) qPCR analysis of calcium-handling genes downregulated and (F) upregulated in $\mathrm{CKO}$ ventricles. $n=4$. All genes in $\mathbf{D}-\mathbf{F}$ are significantly regulated in Med12cKO ventricles. (G) Protein levels in hearts and CMs from CTL and cKO male mice. Ryr, ryanodine receptor; SERCA2a, sarcoplasmic reticulum $\mathrm{Ca}^{2+}$ ATPase 2a; PLN, total phopholamban; CX43, connexin 43. (H) Quantification of Western blots from 6-8 mice. Data are mean \pm SEM. ${ }^{*} P<0.05$ by 2 -tailed Student's $t$ test.

calcium release from the SR through the ryanodine receptor (RyR), leading to CM contraction. Protein levels of RyR were decreased in Med12cKO hearts at P7 and were significantly reduced in CMs isolated from hearts of 8-week-old Med12cKO mice (Figure 3, G and $\mathrm{H}$ ). CM relaxation is regulated by signaling pathways that restore intracellular and $\mathrm{SR} \mathrm{Ca}^{2+}$ to resting concentrations. These pathways converge on SERCA2a, which rapidly pumps $\mathrm{Ca}^{2+}$ into the SR, decreasing the cytosolic intracellular $\mathrm{Ca}^{2+}$ concentration. SERCA2a protein levels were decreased at P7 and 8 weeks in Med12cKO CMs and hearts. Total protein levels of phospholamban (PLN), an inhibitor of SERCA2a, were not significantly changed in P7 or 8 week-old ventricles, but PLN was hyperphosphorylated in Med12cKO CMs and hearts (Figure 3, G and $\mathrm{H}$ ). The phosphorylation of PLN is known to potently relieve its inhibitory effects on SERCA2a, 
A
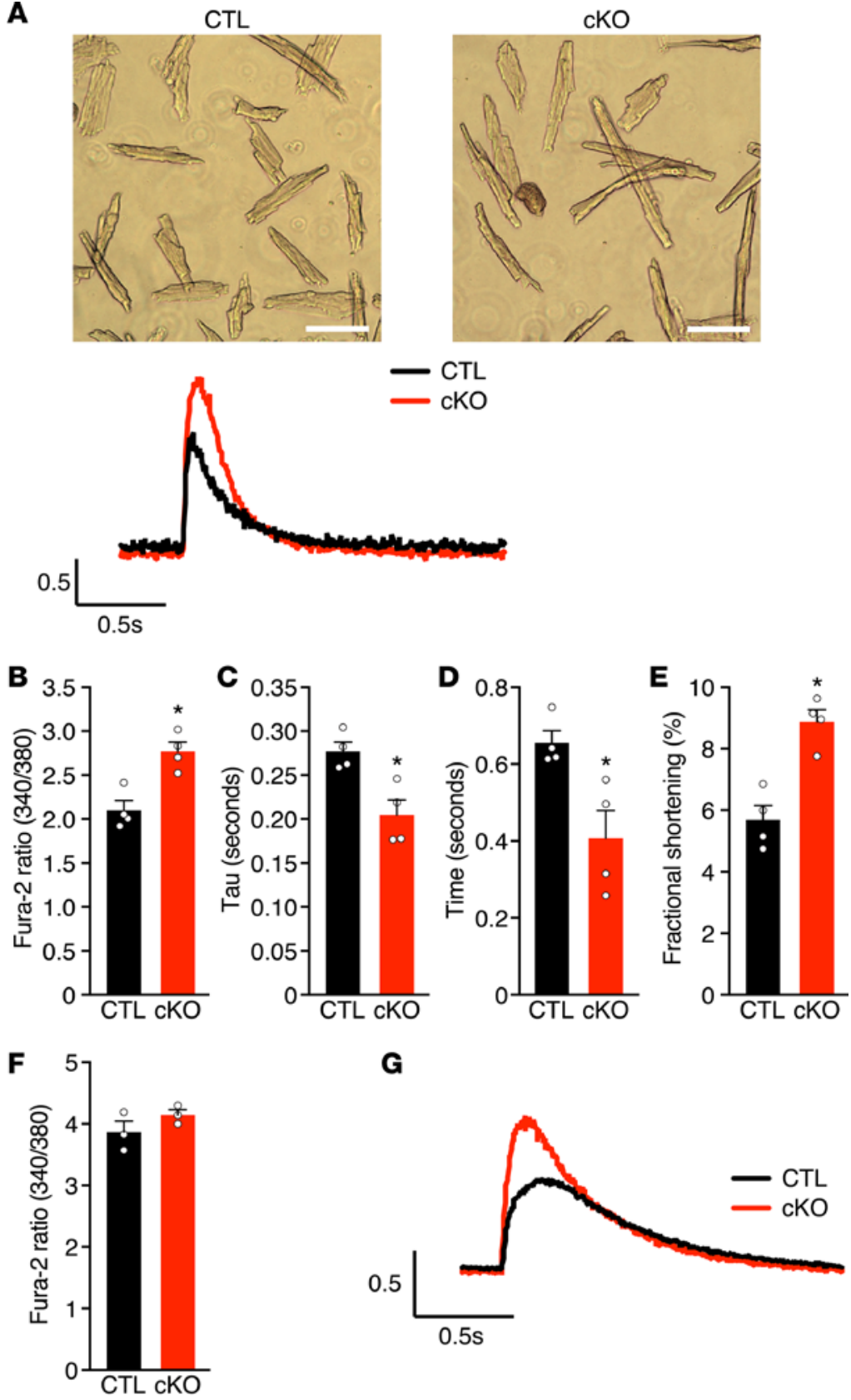

G
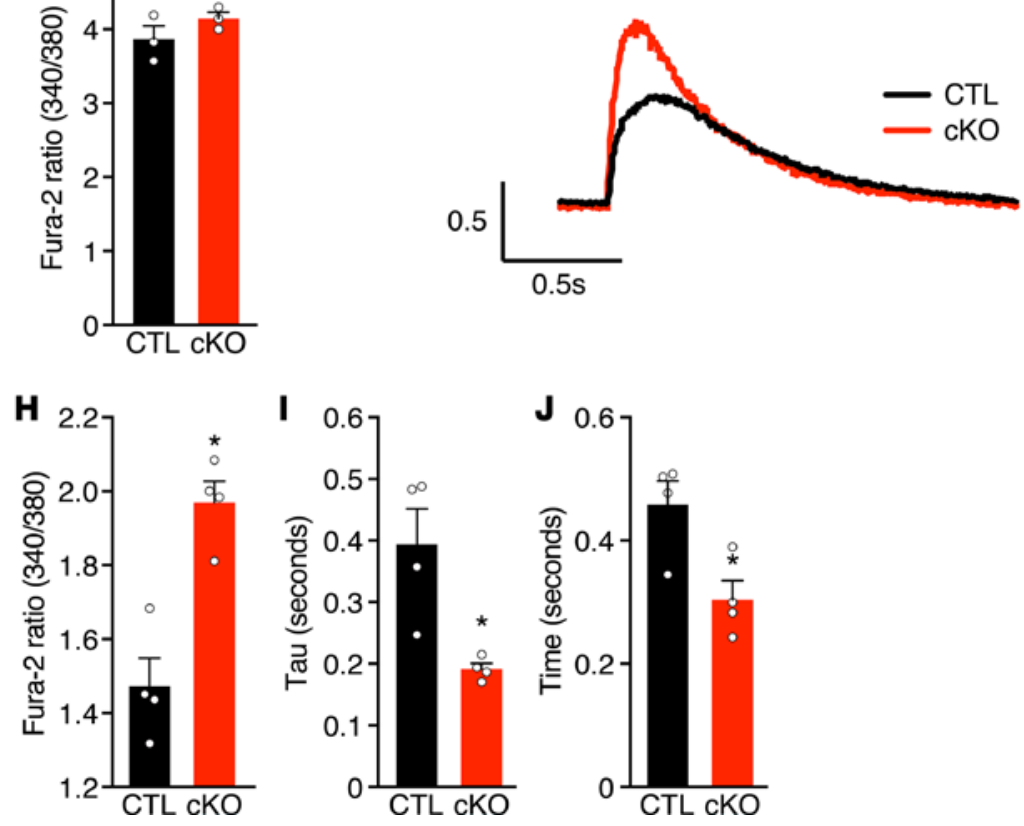

Figure 4. MED12 regulates calcium handling in cardiomyocytes. (A) Adult mouse cardiomyocytes (CM) isolated from 8-week-old male CTL and CKO ventricles and representative calcium tracings. Scale bars: $100 \mu \mathrm{m}$. (B) Peak $\mathrm{Ca}^{2+}$ transients, (C) transient decay rate $(\tau)$, (D) time to $50 \%$ decay, (E) fractional shortening, (F) and SR $\mathrm{Ca}^{2+}$ load in CMs from CTL and cKO adult male mice. (C) Representative calcium transients from neonatal CM isolated from P1 male CTL and cKO ventricles. (H) Peak $\mathrm{Ca}^{2+}$ transients, (I) transient decay rate, and (J) time to $50 \%$ decay in CMs from CTL and cKO neonatal male mice. For $\mathbf{B}-\mathbf{F}$ and $\mathbf{H}-\mathbf{J}, n=16$ cardiomyocytes per heart and $n=$ 3-4 hearts per group. For simplicity, data points are averages of CM calcium measurements from each animal. Data are average \pm SEM. ${ }^{*} P<0.01$ by 2 -tailed Student's $t$ test.

resulting in increased SERCA activity and enhanced calcium cycling. We also observed a significant decrease in the gap junction protein connexin-43 (CX43) in P7 Med12cKO hearts, and they remained decreased at 8 weeks of age in ventricles and in isolated CMs (Figure 3, G and H). Taken together, altered calcium-handling gene and protein levels in $\mathrm{Med} 12 \mathrm{cKO}$ hearts support the hypothesis that MED12 coordinately regulates calcium handling in the heart.

MED12 regulates calcium handling in CMs. To determine the functional consequence of altered calcium-handling gene and protein expression, we measured $\mathrm{Ca}^{2+}$ flux in adult CMs. Loss of Med12 increased peak $\mathrm{Ca}^{2+}$ transients without altering diastolic $\mathrm{Ca}^{2+}$ levels (Figure 4, A and B), indicating that more $\mathrm{Ca}^{2+}$ is released during each contraction-relaxation cycle in Med12cKO CMs. Reuptake of $\mathrm{Ca}^{2+}$ into the SR was accelerated in $M e d 12 c K O \mathrm{CMs}$, as demonstrated by a faster cytosolic $\mathrm{Ca}^{2+}$ transient decay rate $(\tau)$ and reduced duration of the $\mathrm{Ca}^{2+}$ transient (Figure 4, C and D). We also observed an increase in $\mathrm{CM}$ fractional shortening, suggesting enhanced contractility in $\mathrm{Med} 12 \mathrm{CKO} \mathrm{CMs}$ (Figure 4E). To determine whether changes in $\mathrm{Ca}^{2+}$ transients were due to altered SR calcium content, we measured SR $\mathrm{Ca}^{2+}$ load using caffeine. Total SR $\mathrm{Ca}^{2+}$ content is unchanged in Med12cKO CMs (Figure 4F), indicating that it is the cycling of $\mathrm{Ca}^{2+}$ that is altered in adult Med12cKO CMs, rather than the total levels of $\mathrm{Ca}^{2+}$ available in the SR.

Perturbed $\mathrm{Ca}^{2+}$ cycling in CMs could be a cause or consequence of cardiac dysfunction in adult Med$12 c K O$ hearts. Because cardiac function was already decreased in Med12cKO mice at P7 (Figure 1E), we measured $\mathrm{Ca}^{2+}$ flux in CMs isolated from P1 hearts. Similar to adult CMs, loss of Med12 did not alter diastolic $\mathrm{Ca}^{2+}$ levels but increased peak $\mathrm{Ca}^{2+}$ transients, $\mathrm{Ca}^{2+}$ decay rate, and duration of $\mathrm{Ca}^{2+}$ transients in $\mathrm{P} 1$ CMs (Figure 4, G-J). These data demonstrate that altered calcium handling precedes cardiac dysfunction in Med12cKO mice, indicating that it is likely the cause of early-onset DCM. 
MED12 facilitates cooperative transcription of calcium-handling genes in the heart. To gain an understanding of how MED12 regulates gene expression in the heart, we performed Ingenuity Pathway upstream regulator analysis (URA) (29). URA is an unbiased analysis that identifies upstream transcriptional regulators of gene-expression changes based on reports in the literature. The results, displayed as a z-score, infer the activation or inactivation state of the transcription factor. Using URA, we identified likely upstream regulators of genes differentially expressed in $\mathrm{Med} 12 \mathrm{cKO}$ ventricles. Loss of Med12 was predicted to inhibit the activities of transcription factors known to regulate calcium-handling gene expression, including CEBP, MEF2, CREB, EGR1, STAT3, and STAT1 (Figure 5A) (30). With the exception of Egr1, which was downregulated, deletion of $\mathrm{Med} 12 \mathrm{did}$ not affect expression of these transcription factors in the heart (Supplemental Figure 4A). This analysis suggests that MED12 works with these transcription factors to regulate the expression of calcium-handling genes.

Mediator subunits can cooperatively bind multiple transcription factors to regulate gene expression (31), and we investigated whether this was the case for MED12 in the heart. SynoR is a genome-mining software that predicts synonymous regulation of genes based on clusters of transcription factor binding sites (TFBS). We used SynoR to identify genes containing conserved binding sites for the upstream regulators of calcium genes in Med12cKO hearts (CEBP, MEF2, CREB, EGR1, STAT3, and STAT1) (32). Genes with conserved TFBS that were significantly regulated in $\mathrm{Med} 12 c K O$ ventricles were identified and categorized based on the number of TFBS. For example, conserved consensus sequences of all 6 TFs were identified within Atp2a2 (Figure 5C), 5 TFBS were identified in Gja1, 4 TFBS were identified in Ryr2, and 3 TFBS were identified in $P \ln$ (Supplemental Figure 4, B-E). We generated a circos plot to visualize regulatory patterns of genes based on TFBS of likely upstream TFs regulated by cardiac MED12 (Figure 5B). Of the 200 genes significantly regulated in Med12cKO hearts, 22 are associated with at least 4 TFBS for CEBP, MEF2, CREB, EGR1, STAT3, and STAT1. These genes control structural and contractile properties of CMs, and they regulate cardiac development and ion channel activity. Over a third of the genes containing TFBS regulate calcium handling in CMs (Figure 5B and Supplemental Table 2). Identification of conserved consensus sequences for CEBP, MEF2, CREB, EGR1, STAT3, and STAT1 within calcium-handling genes provides additional support that MED12 regulates calcium-handling genes through these transcription factors.

Because most calcium-handling genes are repressed in the absence of MED12 (Figure 3), and they are regulated by transcription factors that are inhibited in the absence of MED12 (Figure 5A), we tested whether MED12 colocalizes with TFBS on calcium-handling genes. We performed ChIP on nuclear fractions of CMs isolated from CTL and Med12cKO ventricles. We probed for the interaction between MED12 and regions of calcium-handling genes identified by SynoR that contain multiple TFBS, such as within Atp2a2 (Figure 5C). PCR was performed on ChIP samples using primers flanking TFBS in Atp2a2, Gja1, Ryr, Pln, and Cacna1d (Supplemental Figure 4, D-G). We found that MED12 interacts with several regions on Atp2a2, Gja1, and Ryr, but not with Pln or Cacnald or in regions devoid of predicted TFBS (negative controls [nc]) (Figure 5D). Additionally, the genomic regions that associate with MED12 on Atp2a2, Gja1, and Ryr contain predicted TFBS for CEBP, MEF2, CREB, EGR1, STAT3, and STAT1 (Supplemental Figure 4, D-G).

MED12 interacts with MEF2. To determine whether MED12 interacts with the predicted URA transcription factors, we performed coimmunoprecipitation. Conserved MEF2 consensus binding sites are located within the amplified regions of Atp2a2, Gja1, and Ryr2 identified by MED12 ChIP (Figure 5D). Therefore, we investigated whether MED12 interacts with MEF2 in CMs. MED12 was immunoprecipitated from nuclear fractions of CMs isolated from CTL and Med12cKO hearts, and the precipitate was probed for MED12 and MEF2. We discovered that MED12 interacts with MEF2 in CTL but not in Med12cKO CMs, suggesting that MED12 can regulate transcription through $\mathrm{MEF} 2$ (Figure $5 \mathrm{E}$ ).

To determine whether MED12 interacts with MEF2 at MEF2 consensus binding sites on calcium-handling genes, we performed ChIP-reChIP experiments on nuclear fractions of CMs isolated from CTL and Med12cKO ventricles. We first immunoprecipitated MED12 from sheared chromatin, as in Figure 5D, and subsequently immunoprecipitated the MED12-chromatin complexes with MEF2. Because MED12 positively interacts with regions of Atp2a2 and Ryr2 at conserved MEF2 binding sites (Figure 5D and Supplemental Figure 4B), we used the same sites as a readout for MED12-MEF2 ChIP-reChIP experiments. MED12 and MEF2 co-occupy the same conserved MEF2 consensus sites on Atp2a2 and Ryr2, but they do not co-occupy conserved CEBP and CREB consensus sites on Atp2a2, conserved STAT1 and STAT3 consensus sites on Ryr2, or regions devoid of predicted TFBS (nc) (Figure 5F). As an additional negative 
A

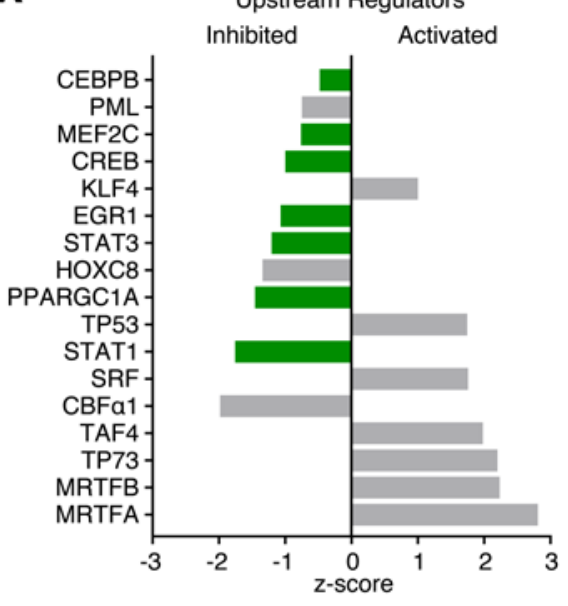

B



Calcium handling

- Structural/contractile

- Cardiac development

- lon channel/regulation Other
C



D

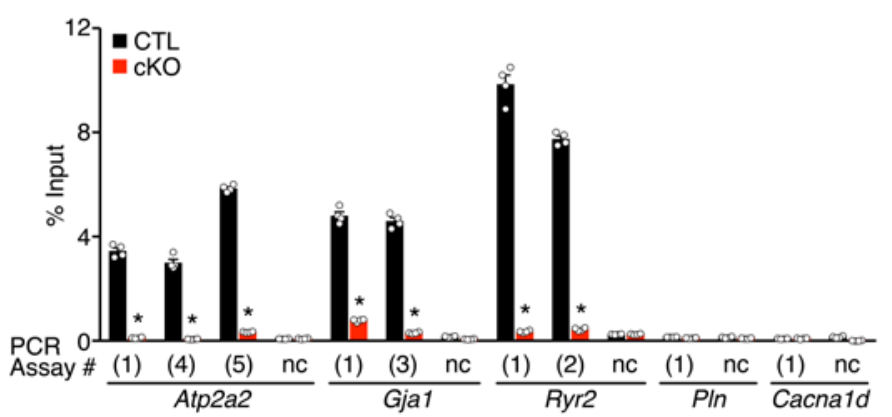

E
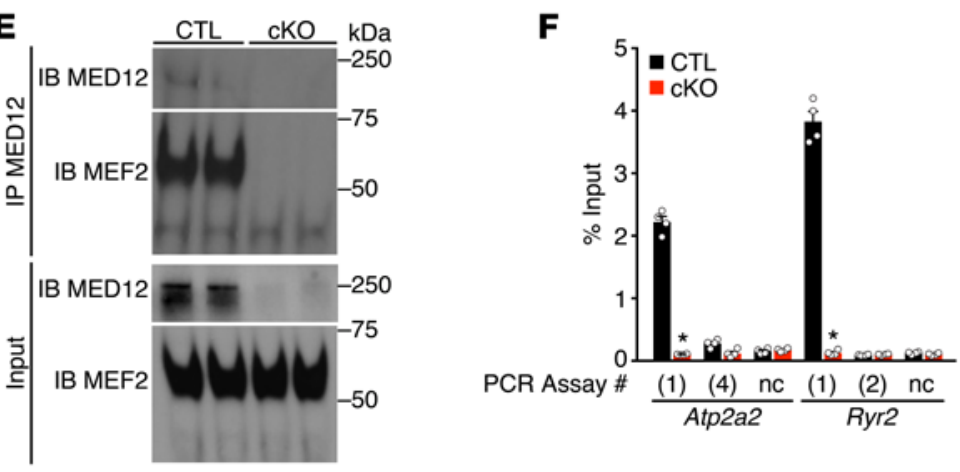

G


MEF2
MEF2
MOtifs

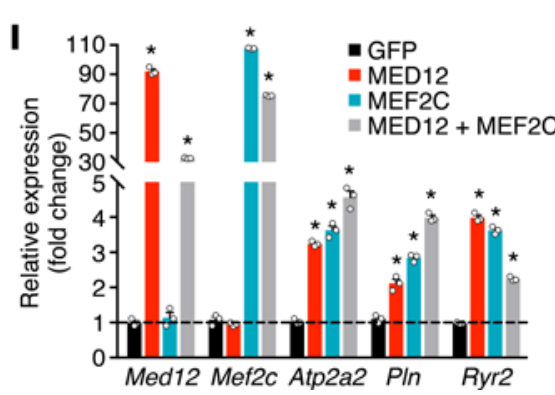

H




Figure 5. MED12 facilitates cooperative transcription of calcium-handling genes in the heart. (A) Upstream regulator analysis (URA) of differentially expressed genes in CTL and CKO ventricles. Bars in green are transcription factors known to regulate calcium-handling genes. (B) Circos plot demonstrating transcription factor binding sites (TFBS) within MED12 target genes. Genes containing TFBS for CEBP, MEF2, CREB, ECR1, STAT3, and STAT1 are categorized by function, and the increased width of connecting ribbons indicates more TFBS (Supplemental Table 2 contains the data used to generate the plot). (C) Chromosomal map of Atp2a2 with TFBS and ChIP-PCR assay primers. (D) MED12 ChIP-PCR results for select calcium-handling genes. PCR assay number corresponds to sequences on chromosomal maps for the indicated genes (see Supplemental Figure 4). Regions devoid of transcription factor binding sites were used as negative controls (nc). $n=4$. (E) Coimmunoprecipitation of MED12 with MEF2 in nuclei isolated from CTL and cKO cardiomyocytes. (F) MED12-MEF2 ChIP-reChIP on Atp2a2 and Ryr2 promoters, PCR assay number corresponds to sequences on chromosomal maps (see $\mathbf{C}$ and Supplemental Figure 4). $n=4$. (C) RNA Polymerase II and MEF2 ChIP-seq signals at the Atp2a2 locus in CTL and cKO ventricles. MEF2 motif locations are shown in green. (H) MEF2 luciferase reporter assays performed in COS-7 cells. $n=3$ independent experiments. Luc, luciferase. (I) Coexpression studies performed in HL-1 cardiomyocytes. $n=3$ independent experiments. Data are average \pm SEM. ${ }^{*} P<0.01$ by 2 -tailed Student's $t$ test $(\mathbf{D}, \mathbf{F}, \mathbf{I})$ or one-way ANOVA with post-hoc Tukey test $(\mathbf{H})$.

control, NFאB did not bind to or co-occupy the conserved CEBP, MEF2, CREB, EGR1, STAT3, or STAT1 consensus sites on Atp2a2, Gja1, Ryr2, Pln, or Cacna1d with MED12 (Supplemental Figure 4, F and G). Our results confirm previous ChIP-seq data in HL-1 CMs, demonstrating that ChIP peaks from exogenously overexpressed MEF2 are detected within Atp2a2 and Ryr2 (33). To further define MEF2 binding sites in CTL and Med12cKO ventricles, we performed MEF2 ChIP-seq on endogenous MEF2. We validated the MEF2 ChIP-seq peaks by performing de novo motif discovery using the top 8,000 peaks identified in ventricular samples (34), and the top motif identified has been previously reported for MEF2 (Supplemental Figure 5A). MEF2 peaks were identified within many of the calcium-handling genes regulated by MED12, including Atp2a2 (Figure 5G), Ryr2, Pln, and Cacna1d (Supplemental Figure 5). We did not observe any significant differences in MEF2 binding in the presence or absence of MED12. These data demonstrate that MED12 is not required for MEF2-DNA interaction.

Given that the Mediator kinase submodule regulates RNA Pol II recruitment and, therefore, transcriptional activation, we investigated the specific role of MED12 and its effect on Pol II-DNA binding. We performed RNA Pol II ChIP-seq on endogenously expressed Pol II in ventricles from CTL and Med12cKO mice. We did not observe significant differences in RNA Pol II binding in the presence or absence of MED12. Specific results are shown for the calcium genes of interest (Figure 5G and Supplemental Figure 5). These data suggest that MED12 is not required for RNA Pol II recruitment to transcription start sites in the heart.

MED12 directly stimulates MEF2 transcriptional activity. To investigate whether MED12 activates MEF2 transcriptional activity, we performed luciferase reporter assays with a synthetic 3XMEF2 luciferase promoter, which is activated by MEF2 (35). Interestingly, MED12 activated endogenous MEF2 transcriptional activity in COS-7 cells, shown by a 2-fold increase in normalized luciferase activity compared with basal luciferase activity (HA-VP16). Luciferase activity was increased 50-fold over basal luciferase activity in the presence of active MEF2 (MEF2 HA-VP16). The addition of MED12 increased MEF2 transcriptional activity by 6 -fold, and MED12 and MEF2 synergistically activated luciferase activity by 340 -fold over basal luciferase activity (Figure $5 \mathrm{H}$ ).

Our in vivo data demonstrate that MED12 is necessary to maintain normal expression of calcium-handling genes in the heart. To determine whether MED12 is sufficient to regulate calcium-handling genes through MEF2, we expressed MED12 and MEF2 in HL-1 CMs. MED12 and MEF2 alone increased expression of Atp2a2, Pln, and Ryr2 in CMs. Coexpression of MED12 and MEF2 had an additive effect on the expression of Atp2a2 and Pln, but not Ryr2 (Figure 5I). Collectively, our studies demonstrate that MED12 is required to maintain normal cardiac function by transcriptionally regulating calcium-handling genes through interaction with transcription factors such as MEF2.

\section{Discussion}

In this study, we investigated the function of MED12 in the postnatal heart. We demonstrate that MED12 is required to maintain cardiac function by regulating calcium cycling in CMs. MED12 regulates gene expression, in part, by coordinating transcription through multiple transcription factors known to regulate calcium-handling genes, including MEF2. Our results support a role for MED12 as a transcriptional regulator that controls the transcriptional network of calcium-handling genes in the heart.

Cardiac contractility is controlled at the molecular level by calcium flux. Release and reuptake of intracellular calcium drives contraction and relaxation, and this process is tightly regulated in CMs (Figure 6). Disruption of calcium-handling genes results in altered cardiac contractility. For example, reduced RyR 


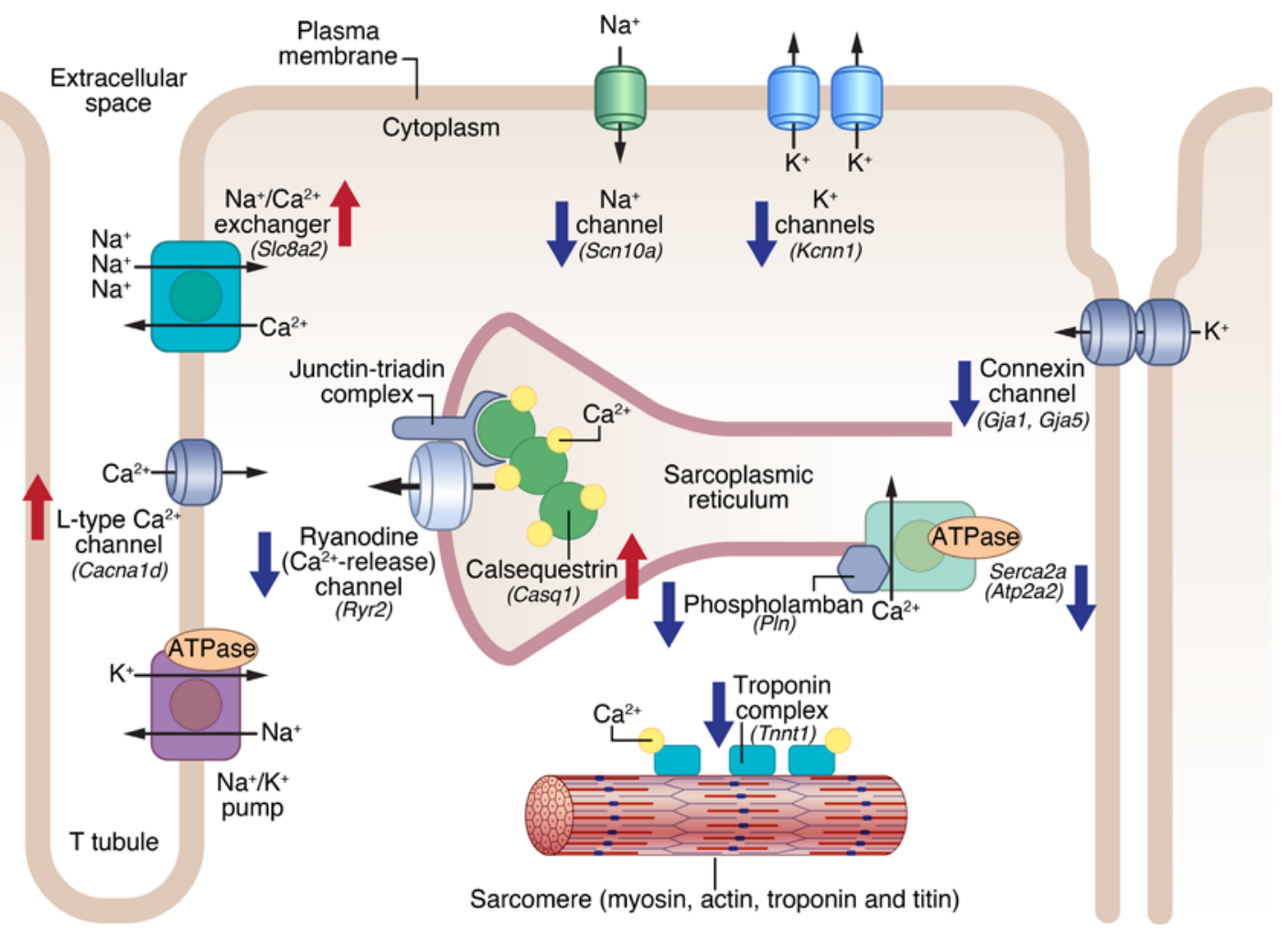

Figure 6. MED12 coordinates calcium cycling in cardiomyocytes. Proper $\mathrm{Ca}^{2+}$ cycling and $\mathrm{Ca}^{2+}$-dependent signaling is required to maintain normal cardiac contractility. Excitation-contraction (EC) coupling is initiated by an action potential which depolarizes the sarcolemma by rapid sodium influx through sodium channels ( $\mathrm{C}$ cn10a). Depolarization activates voltage-gated L-type $\mathrm{Ca}^{2+}$ channels, and $\mathrm{Ca}^{2+}$ influx triggers calcium-induced calcium release from the SR via the ryanodine receptor (Ryr2). Rapid release of $\mathrm{Ca}^{2+}$ from the SR increases free intracellular $\mathrm{Ca}^{2+}$, enabling muscle contraction. Gap junctions comprised of connexin complexes (Gja1 and Gja5) connect neighboring cells and facilitate the dispersion of action potentials throughout the heart. Cardiomyocyte relaxation is regulated by signaling pathways that restore intracellular and SR $\mathrm{Ca}^{2+}$ to resting concentrations. Ca ${ }^{2+}-$ activated kinases phosphorylate phospholamban (PIn), relieving its repression on SERCA2a (Atp2a2). Consequently, SERCA2a rapidly imports Ca ${ }^{2+}$ into the SR, decreasing the intracellular $\mathrm{Ca}^{2+}$ concentration. $\mathrm{Na}^{+} / \mathrm{Ca}^{2+}$ exchangers (NCX) (S/c8a2) are allosterically activated by $\mathrm{Ca}^{2+}$ and aid in restoring resting Ca ${ }^{2+}$ concentrations, and decreased cytosolic $\mathrm{Ca}^{2+}$ leads to relaxation of the sarcomere. Genes downregulated in Med12cKO hearts are denoted by a blue downward arrow and genes upregulated are denoted by a red upward arrow.

protein levels in adult mouse hearts decreases heart rate and cardiac function, similar to Med12cKO mice (5). CMs from PLN-null $\left(\mathrm{Pln}^{-/}\right)$mice display increased $\mathrm{Ca}^{2+}$ transient amplitude and faster $\mathrm{Ca}^{2+}$ decay rate, as seen in $\operatorname{Med} 12 c K O \mathrm{CMs}(6)$. SERCA2a-null (Atp2a2 $2^{-/}$) mice are embryonically lethal, but heterozygous mice have impaired intracellular calcium homeostasis and decreased contractile function (7). It is also interesting to note that Egr1, a transcription factor predicted as an upstream regulator in $\mathrm{Med} 12 \mathrm{cKO}$ hearts, is the only transcription factor that is significantly downregulated in Med12cKO hearts (Figure 5A). Egr $^{-/}$mice have decreased levels of RyR, SERCA2a, and PLN, similar to Med12cKO mice (Figure 3) (36). Indeed, many characteristics of $\mathrm{Med} 12 \mathrm{cKO}$ hearts mimic those reported for calcineurin transgenic mice. Calcineurin transgenic mice display significant cardiac dysfunction, while isolated transgenic CMs have faster cytosolic calcium decay rates and duration of calcium transients. Additionally, PLN phosphorylation is enhanced in transgenic mice, and CX43 is also significantly decreased (37). The phenotypic similarities between these mouse models and Med12cKO mice provide additional evidence that MED12 is indeed a regulator of calcium-handling genes in the heart.

One way that MED12 regulates calcium-handling genes in CMs is by stimulating the transcriptional activity of MEF2 (Figure 5). We demonstrated that MED12 and MEF2 co-occupy MEF2 consensus sites within several genes, and that MED12 and MEF2 interact in CMs. Overexpression of MEF2 and MED12 also increases expression of calcium-handling genes (Figure 5). Under basal conditions, MEF2 activity is repressed by class II histone deacetylases (HDACs), but HDACs are posttranslationally modified by $\mathrm{Ca}^{2+}$-responsive kinases, consequently relieving repression of MEF2 transcriptional activity (38, 39). MEF2 also cooperates with other transcription factors to drive expression of target genes (40). Our data suggest 
that, in CMs, MED12 is also an important coregulator of MEF2 transcriptional activity and may help recruit other transcription factors to regulate MEF2 target genes. Interestingly, MED12 and MEF2 have been independently shown to activate transcription of Wnt and sonic hedgehog target genes in Drosophila (41-43). In the mammalian heart, however, MED12 and MEF2 seem to function as regulators of calcium-handling genes. In future work, it will be important to determine the mechanism by which MED12 regulates MEF2 transcriptional activity.

Very little is known about the specific role of Mediator subunits in the heart $(44,45)$. In an effort to understand the physiological functions of Mediator, mouse models of several Mediator subunits have been generated. Mice with missense mutations in Med30 develop DCM after weaning. Early lethality in these mice was linked to a decline in mitochondrial function, likely due to decreased expression of genes involved in oxidative phosphorylation in the heart (46). We recently demonstrated that cardiac MED13 regulates metabolic gene expression in the heart. Increased expression of Med13 enhances metabolism and protects mice from diet-induced obesity, and we have evidence that circulating factors in these mice regulate body weight $(15,16)$. MED1 regulates development in multiple organs, and Med1-null and hypomorphic mice, both of which are embryonically lethal, have cardiac developmental defects $(47,48)$. It was recently reported by two independent groups that cardiac-specific deletion of Med 1 leads to severe DCM and premature death in mice. Although many calcium-handling genes were downregulated in the $\mathrm{Med}^{-1}$ heart, they were measured after the onset of DCM, which has been associated with decreased calcium-handling gene expression, as discussed above $(49,50)$.

Mutations in several Mediator subunits have been linked to congenital heart disease. Patients with DiGeorge syndrome have hemizygous deletions within Med15 and often present with congenital heart defects (51), and missense mutations in Med13l have also been linked to patients with congenital heart defects (52). Interestingly, some male patients diagnosed with FG/Opitz-Kaveggia syndrome have a single missense mutation in Med12 and suffer from congenital heart defects $(53,54)$. Because Med12 is on the $\mathrm{X}$-chromosome, clinical reports of patients with congenital Med12 mutations have been males, likely due to the severity of disease symptoms in male patients. Recently, however, somatic mutations in Med12 have been reported in several types of cancer in females, including uterine leiomyoma and breast fibroadenoma $(55,56)$. Additionally, our data suggest that random inactivation of Med12 in female hearts causes cardiac dysfunction ( $\alpha M h c-C r e^{+} M e d 12^{f /+}$, Supplemental Figure 1). These studies provide evidence that MED12 is also important in regulating non-X-linked diseases. Given the prominent role of Mediator in transcriptional regulation in all cell types, it is likely that these discoveries are the first of many to identify diseases associated with genetic mutations in Med12. It will be interesting to investigate whether loss of function mutations in Med12 might also contribute to DCM and heart failure in humans.

The Mediator kinase submodule was originally thought to act as a transcriptional repressor, but there is increasing evidence that kinase subunits, particularly MED12, activate transcription. In zebrafish, for example, mutant Med12 decreases Sox4-dependent gene expression during pancreas development, and in liver, mutant Med12 decreases HNF-4-dependent gene expression. Consequently, mutant Med12 prevents pancreatic $\alpha$ cell and hepatocyte differentiation, although through different mechanisms (22). Similarly, in another zebrafish model, mutant Med12 causes defects in craniofacial development, and MED12 is required for Sox9-dependent gene expression in bone and cartilage (57). In human embryonic kidney (HEK293) cells, MED12 interacts with $\beta$-catenin to positively regulate Wnt-mediated gene expression (58), and in Drosophila, MED12 is required for transcriptional activation of Wnt target genes (41).

MED12 is ubiquitously expressed in all cell types but regulates gene expression in a cell-specific manner, presumably by synergizing with different transcription factors and chromatin modifiers. Indeed, MED12 is a corepressor of REST, which acts to silence neuronal-specific gene expression (21), and developmental transcription factors — such as $\beta$-catenin, GLI1, and Nanog — interact with MED12 in differing cell types to help determine cell fate $(42,58,59)$. Furthermore, comparison of MED12 ChIP-seq peaks from mouse embryonic stem cells, mouse embryonic fibroblasts (60), and primary human or mouse CD34 ${ }^{+}$hematopoietic stem/progenitor cells (61) show some overlap, but MED12 localizes to specific chromatin regions in different cell types. There is also evidence that MED12 has opposing functions in different cell types. For example, as mentioned above, MED12 positively regulates Wnt target genes in HEK293 cells and in Drosophila $(41,58)$, but in Caenorhabditis elegans (C. elegans), MED12 seems to be an inhibitor of $\mathrm{Wnt} / \beta$-catenin-mediated gene expression $(62,63)$. We previously reported opposing metabolic functions of MED13 in cardiac and skeletal muscle, yet another example of cell-specific functions 
of Mediator subunits (64). Additionally, loss of Med23 facilitates smooth muscle cell differentiation but represses adipocyte differentiation by regulating different sets of genes in a cell-dependent manner (65).

Here, we focused on determining a role for MED12 in the heart. We found that MED12 is a transcriptional regulator of calcium-handling genes in CMs. Whether MED12 regulates calcium-handling genes in other tissues, such as skeletal muscle, is currently under investigation. Future work will investigate the roles of other transcription factors that interact with MED12 in the heart. These studies will reveal additional transcriptional networks regulated by MED12 that are important to maintain cardiac function.

\section{Methods}

Animals. Animals were housed in a pathogen-free barrier facility with a 12-hour light/dark cycle and maintained on standard chow (2916 Teklad Global). The generation of Med12 $2^{f / f l}$ mice is published (24). Med $12 c K O$ were generated by crossing female $M e d 12^{f / f l}$ mice to male mice expressing the $\alpha M h c$-Cre transgene (26). Med12 is located on the X chromosome, and all $\alpha M h c-C r e^{+}$male mice with a single-floxed Med12 allele $\left(\alpha M h c-C r e^{+} \mathrm{Med} 12^{f / y}\right)$ are abbreviated as $\mathrm{Med} 12 \mathrm{KO}$.

Histology. Hearts were isolated and fixed in $4 \%$ (vol/vol) paraformaldehyde in PBS for 48 hours at $4^{\circ} \mathrm{C}$ with gentle shaking. Hearts were dehydrated, embedded in paraffin, and sectioned. Heart sections were stained with H\&E, Masson's trichrome, TUNEL, propidium iodide, and Ki-67 using standard procedures. Heart sections were costained with cardiac Troponin T (MA5-12960, Thermo Fisher Scientific) to identify CMs.

Transmission electron microscopy. Hearts were fixed by perfusion with $4 \%$ paraformaldehyde and $1 \%$ glutaraldehyde in $0.1 \mathrm{M}$ sodium cacodylate buffer ( $\mathrm{pH}$ 7.4). Samples were processed by the University of Texas Southwestern Medical Center Electron Microscopy Core facility. Briefly, fixed tissues were post-fixed, stained, dehydrated, and embedded in EMbed-812 resin (Thermo Fisher Scientific). Tissue sections were cut and post-stained, and images were acquired on a FEI Tecnai $\mathrm{G}^{2}$ Spirit TEM.

Transthoracic echocardiography. Cardiac function and heart dimensions were determined by 2-dimensional echocardiography using a Visual Sonics Vevo 2100 Ultrasound (Visual Sonics) on nonanesthetized mice. M-mode tracings were used to measure anterior and posterior wall thicknesses at end diastole and end systole. LVID was measured as the largest anteroposterior diameter in either diastole (LVIDD) or systole (LVIDS). A single observer blinded to mouse genotypes performed echocardiography and data analysis. Fractional shortening (FS) was calculated according to the following formula: FS(\%) $=([\mathrm{LVIDd}$ - LVIDs]/LVIDd $\times 100$. Ejection fraction $(\mathrm{EF} \%)$ was calculated by: $\mathrm{EF}(\%)=(\mathrm{EDV}-\mathrm{ESV}) / \mathrm{EDV}$, where ESV represents end systolic volume and EDV represents end diastolic volume.

Electrocardiography. ECG was performed on sedated adult male mice (6 weeks of age) using Accutac Diaphoretic ECG Electrodes (ConMed Corp.). Electrodes were attached to all 4 limbs, and leads I, II, III, aVR, aVL, and aVF were recorded using PageWriter XLs (Hewlett Packard). Traces were recorded using identical settings for CTL and Med12cKO mice (50 mm/s; $20 \mathrm{~mm} / \mathrm{mV})$.

$R N A$ extraction and quantitative PCR analysis. Total RNA was isolated from both ventricles of CTL and Med12cKO mice using TRIzol according to the manufacturer's instructions (Thermo Fisher Scientific). cDNA was synthesized using iScript Reverse Transcription Supermix with random primers (Bio-Rad). Gene expression was analyzed by quantitative PCR (qPCR) using KAPA SYBR FAST (Kapa Biosystems) on a 7900HT Fast Real-Time PCR machine (Applied Biosystems). Expression amount was normalized to $18 S$ mRNA and was represented as fold change.

RNA deep sequencing analysis. Total RNA was isolated from both ventricles of male P1 CTL and Med12cKO mice using TRIzol according to the manufacturer's instructions. Illumina RNA-Seq was performed by the University of Texas Southwestern Microarray Core Facility. Quality assessment of the RNA-Seq data was performed using NGS-QC-Toolkit (v 2.3.3) with default settings (66). Quality filtered reads were aligned to the mouse reference genome GRCm38 (mm10) using the Tophat2 (v 2.0.0) aligner with default settings (67). The R package DESeq2 (v 1.6.3) was used for differential gene expression analysis (68). Read counts obtained from featureCounts were normalized by taking the median of each gene count across samples and dividing each sample gene count by the relative ratio of library sizes between the calculated median and sample size (69). The average normalized expression values of the samples were used to calculate fold change and $P$ values. Cutoff values of fold change greater than 1.5 and $P<$ 0.05 were then used to select for differentially expressed genes between sample group comparisons.

Significant pathway enrichment analysis was performed using Ingenuity Pathways Analysis (Ingenuity Systems). Fisher's exact test was used to calculate the p-value and determine the probability that each 
biological function was enriched in the dataset due to chance alone. Statistically significant biological pathways were then identified by selection for pathways with $P<0.05$.

The DAVID gene functional annotation and classification tool was used to annotate the list of differentially expressed genes with respective GO terms and GO enrichment analysis for molecular and biological functional categories was performed. Functional GO groups were selected for significance by using a $P$ value cutoff of $1 \%$ (70). For both IPA and GO enrichment analysis, nonredundant pathways and functions are reported.

To identify upstream transcriptional regulators of gene expression in the analyzed dataset, IPA URA was performed, and activation z-scores were computed.

In silico analysis. The SynoR tool was used to predict genes that are cooperatively regulated by multiple transcription factors (32). Multiple groups of transcription factors, predicted by IPA to regulate gene expression in $\mathrm{Med} 12 \mathrm{KOO}$ hearts, were analyzed to identify the genes they synonymously regulate. Regulation of these genes was then biochemically tested using ChIP analysis.

Adult mouse CM isolation. Adult mouse hearts were rapidly excised, and the aorta was cannulated on a constant-flow Langendorff perfusion apparatus. Hearts were digested with perfused Tyrode's solution (10 mM glucose, $5 \mathrm{mM}$ HEPES, $5.4 \mathrm{mM} \mathrm{KCl}, 1.2 \mathrm{mM} \mathrm{MgCl}$, $150 \mathrm{mM} \mathrm{NaCl}, 2 \mathrm{mM}$ sodium pyruvate, $\mathrm{pH}$ 7.4) (Sigma-Aldrich) containing Liberase $(0.25 \mathrm{mg} / \mathrm{ml})$ (Roche Diagnostics), and the ventricles were minced, filtered, and equilibrated with Tyrode's solution containing $1 \mathrm{mM} \mathrm{CaCl}_{2}$ (Sigma-Aldrich) and BSA (Gemini) at room temperature (15).

Neonatal mouse CM isolation. Neonatal mouse hearts were extracted, and neonatal mouse CMs were isolated from both ventricles using the Isolation System for Neonatal Rat/Mouse CMs (Cellutron) according to the manufacturer's instructions.

CM intracellular calcium and contractility measurements. Adult and neonatal CMs were loaded with 0.5 $\mu \mathrm{M}$ Fura-2-AM (Molecular Probes) and placed in a heated chamber $\left(37^{\circ} \mathrm{C}\right)$ on the stage of an inverted microscope. The chamber was perfused with Tyrode's solution containing $\mathrm{CaCl}_{2}(1.8 \mathrm{mM})$, glucose $(10$ $\mathrm{mM}), \operatorname{HEPES}(5 \mathrm{mM}), \mathrm{KCl}(5.4 \mathrm{mM}), \mathrm{MgCl}_{2}(1.2 \mathrm{mM}), \mathrm{NaCl}(150 \mathrm{mM})$, and sodium pyruvate $(2 \mathrm{mM})$ (pH 7.4) (Sigma-Aldrich). An IonOptix Myocyte Calcium and Contractility System was used to pace CMs at $0.5 \mathrm{~Hz}$ with a MyoPacer field stimulator (IonOptics), and changes in intracellular $\mathrm{Ca}^{2+}$ levels were monitored using Fura-2 dual-excitation (340/380 nm) (Molecular Probes), single emission (510 $\mathrm{nm}$ ) ratiometric imaging. For SR load measurements, field stimulation was stopped and $10 \mathrm{mM}$ caffeine (Sigma-Aldrich) was rapidly applied to the cells. Peak $\mathrm{Ca}^{2+}$ transient amplitude, $\tau$, and the duration of the $\mathrm{Ca}^{2+}$ transient were determined using IonWizard 6.0 analysis software (IonOptix) (71). Adult CM contractility measurements were made using sarcomere length (SarcLen) parameters and processed with IonWizard 6.0 software.

Nuclear fractionation and immunoprecipitation. Nuclear extracts were isolated from CMs using the NE-PER Nuclear and Cytoplasmic Extraction Kit (Pierce) according to manufacturer's instructions. Nuclear extracts were incubated with a MED12 antibody (A300-774A, Bethyl Laboratories) overnight and immunoprecipitated the following day using Dynabeads (Thermo Fisher Scientific) according the manufacturer's protocol. After excessive washing, the bound protein was boiled off the Dynabeads and resolved on a $4 \%-20 \%$ precast polyacrylamide gel.

ChIP and ChIP-reChIP. Nuclear extracts isolated from adult CMs were used to perform ChIP and ChIP-reChIP. Nuclei were crosslinked, with formaldehyde (final concentration of $2 \%$ ) for 60 minutes at room temperature with gentle shaking (72). The cross-linking reaction was terminated by adding glycine for 10 minutes, and crosslinking was reversed by overnight incubation at $65^{\circ} \mathrm{C}$. Sheared chromatin was immunoprecipitated with an antibody against MED12 (A300-774A, Bethyl Laboratories) or NFкB p50 (sc-1190 X, Santa Cruz Biotechnology Inc.) (73), and DNA was isolated and analyzed by PCR with primers flanking binding sites for the indicated TFBS. For ChIP-reChIP, sheared chromatin was immunoprecipitated with an antibody against MED12 (A300-774A, Bethyl Laboratories), followed by immunoprecipitation with an antibody against MEF2 (sc313 X, Santa Cruz Biotechnology Inc.) or NFkB p50 (sc-1190 X, Santa Cruz Biotechnology Inc.), and DNA was isolated and analyzed by PCR with primers flanking binding sites for the indicated TFBS or regions devoid of TFBS as nc. Results are normalized to input and reported as percent input.

ChIP-seq. ChIP was performed as described above, and ChIP-seq libraries were constructed using the Kapa Hyper Prep Kit (KK8500, Roche) according to the manufacturer's instruction. Raw reads were 
mapped using Bowtie2 to obtain about 50 million uniquely mapped single end reads per library (74). Peak calling was performed by MACS/MACS2 (75). The resulting output files included the peak list in BED format, ChIP signal in bedgraph format, and mapped reads in BAM format. All files were subjected to integrative in-depth analysis with published data and data generated by the Encode project using the Bioconductor software project site (76). De novo motif discovery in MEF2 ChIP-seq peak regions were identified using Regulatory Sequence Analysis Tools (RSAT) (34). ChIP-seq data tracks for MEF2 and RNA Poll II were visualized using Integrative Genomics Viewer available online from the Broad Institute, and figures were generated using this software (77).

Immunoblotting. Protein was isolated from homogenized ventricles in modified RIPA buffer. Protein $(10 \mu \mathrm{g})$ was loaded per sample, and proteins were detected using the following antibodies: MED12 (A300774A, Bethyl Laboratories), RyR (C3-33, Pierce), SERCA2a (2A7-A1, Pierce), total PLN (2D12, Pierce), pSer16 PLN (A010-12, Badrilla), pThr17 PLN (A010-13, Badrilla), Connexin 43 (ab11370, Abcam), MEF2 (sc-313, Santa Cruz Biotechnology Inc.), and GAPDH (MAB374, Millipore).

Cell culture, transfection, and luciferase reporter assays. For luciferase reporter assays, COS-7 cells (CRL-1651; ATCC) were grown in DMEM containing 10\% FBS. Transfections were performed with FuGENE 6 Transfection Reagent (Promega) according to the manufacturer's instructions. Cells were plated in 6-well dishes, and after 12 hours of incubation, transfections were performed. Plasmid DNA (100 ng of total) was used for all conditions. All luciferase plasmids have been previously described (35, 78). Med12 was cloned into p3XFLAG-CMV-10 vector, and a CMV promoter-driven LacZ expression plasmid was included for all transfections as an internal control. Cells were lysed 48 hours after transfection, and luciferase activity (Promega) and $\beta$-galactosidase activity (Invitrogen) were determined according to the manufacturer's instructions.

HL-1 cells, a CM-derived cell line (79), were a gift from the WC Claycomb laboratory. HL-1 CMs were cultured according to the protocol and plated in 6-well dishes, and after 12 hours of incubation, transfections were performed with FuGENE 6 Transfection Reagent (Promega) according to the manufacturer's instructions. Mef $2 \mathrm{C}$ was cloned into a pcDNA vector containing a CMV promoter, the same Med12 plasmid was used as described for the luciferase assays, and a GFP plasmid was used as a control. GFP fluorescence was detected in HL-1 CMs 12 hours after transfection. Cells were lysed 24 hours after transfection, total RNA was isolated, gene expression was analyzed by qPCR, and expression was normalized to $18 S$ mRNA. Data is represented as fold change compared with GFP transfected cells.

Statistics. All data are expressed as mean \pm SEM. Two-tailed Student's $t$ test or one-way ANOVA with post hoc Tukey's test was performed to determine statistical significance, and the analysis is specified in the figure legends. A $P<0.05$ was considered statistically significant.

Study approval. All animal procedures were approved by the IACUC at University of Texas Southwestern Medical Center.

\section{Author contributions}

$\mathrm{KKB}$ performed the majority of the experiments, analyzed the data, and wrote the paper. CAM conducted the calcium transient studies in CMs and helped write the paper. SMD performed initial characterization of male mice. WY performed and analyzed the ChIP-seq experiments. BC analyzed the RNA-Seq data and performed all bioinformatic analysis. NB performed the echocardiography experiments. RBD and ENO directed the study and helped write the paper. HS provided the Med12-floxed mice.

\section{Acknowledgments}

We thank the members of the ENO laboratory for helpful discussions, Jose Cabrera for help with images, and Wei Tan for echocardiography assistance. We thank John Shelton, James Richardson, the University of Texas Southwestern Histology Core for assistance with histology, and the University of Texas Southwestern Electron Microscopy Core for assistance with EMs. We also thank the University of Texas Southwestern Genomics and Microarray Core for assistance with RNA-Seq. This work was supported by grants from the NIH (HL-077439, HL-130253, DK-099653, AR-067294, and HL-11165), Foundation Leducq Networks of Excellence (14CVD04 to ENO), and the Robert A. Welch Foundation (1-0025 to ENO), NHLBI (1F32HL129674 to CAM), and the American Heart Association (16POST31100009 to KKB). 
Address correspondence to: Eric N. Olson, University of Texas Southwestern Medical Center, 5323 Harry Hines Boulevard, Dallas, Texas 75390, USA. Phone: 214.648.1187; Email: eric.olson@utsouthwestern.edu.

1. Writing Group Members, et al. Heart Disease and Stroke Statistics-2016 Update: A Report From the American Heart Association. Circulation. 2016;133(4):e38-360.

2. Marks AR. Calcium cycling proteins and heart failure: mechanisms and therapeutics. J Clin Invest. 2013;123(1):46-52.

3. Bers DM. Cardiac sarcoplasmic reticulum calcium leak: basis and roles in cardiac dysfunction. Annu Rev Physiol. 2014;76:107-127.

4. Gorski PA, Ceholski DK, Hajjar RJ. Altered myocardial calcium cycling and energetics in heart failure--a rational approach for disease treatment. Cell Metab. 2015;21(2):183-194.

5. Bround MJ, et al. Cardiac ryanodine receptors control heart rate and rhythmicity in adult mice. Cardiovasc Res. 2012;96(3):372-380.

6. Wolska BM, Stojanovic MO, Luo W, Kranias EG, Solaro RJ. Effect of ablation of phospholamban on dynamics of cardiac myocyte contraction and intracellular Ca2+. Am J Physiol. 1996;271(1 Pt 1):C391-C397.

7. Periasamy $\mathrm{M}$, et al. Impaired cardiac performance in heterozygous mice with a null mutation in the sarco(endo)plasmic reticulum Ca2+-ATPase isoform 2 (SERCA2) gene. J Biol Chem. 1999;274(4):2556-2562.

8. Sato Y, et al. Cardiac-specific overexpression of mouse cardiac calsequestrin is associated with depressed cardiovascular function and hypertrophy in transgenic mice. J Biol Chem. 1998;273(43):28470-28477.

9. Verheule S, Kaese S. Connexin diversity in the heart: insights from transgenic mouse models. Front Pharmacol. 2013;4:81.

10. Conaway RC, Conaway JW. Function and regulation of the Mediator complex. Curr Opin Genet Dev. 2011;21(2):225-230.

11. Allen BL, Taatjes DJ. The Mediator complex: a central integrator of transcription. Nat Rev Mol Cell Biol. 2015;16(3):155-166.

12. Sato $\mathrm{S}$, et al. A set of consensus mammalian mediator subunits identified by multidimensional protein identification technology. Mol Cell. 2004;14(5):685-691.

13. Näär AM, Taatjes DJ, Zhai W, Nogales E, Tjian R. Human CRSP interacts with RNA polymerase II CTD and adopts a specific CTD-bound conformation. Genes Dev. 2002;16(11):1339-1344.

14. Belakavadi M, Fondell JD. Cyclin-dependent kinase 8 positively cooperates with Mediator to promote thyroid hormone receptor-dependent transcriptional activation. Mol Cell Biol. 2010;30(10):2437-2448.

15. Baskin KK, et al. MED13-dependent signaling from the heart confers leanness by enhancing metabolism in adipose tissue and liver. EMBO Mol Med. 2014;6(12):1610-1621.

16. Grueter CE, et al. A cardiac microRNA governs systemic energy homeostasis by regulation of MED13. Cell. 2012;149(3):671-683

17. Risheg H, et al. A recurrent mutation in MED12 leading to R961W causes Opitz-Kaveggia syndrome. Nat Genet. 2007;39(4):451-453.

18. Schwartz CE, et al. The original Lujan syndrome family has a novel missense mutation (p.N1007S) in the MED12 gene. J Med Genet. 2007;44(7):472-477.

19. Vulto-van Silfhout AT, et al. Mutations in MED12 cause X-linked Ohdo syndrome. Am J Hum Genet. 2013;92(3):401-406.

20. Treisman J. Drosophila homologues of the transcriptional coactivation complex subunits TRAP240 and TRAP230 are required for identical processes in eye-antennal disc development. Development. 2001;128(4):603-615.

21. Ding N, et al. Mediator links epigenetic silencing of neuronal gene expression with x-linked mental retardation. Mol Cell. 2008;31(3):347-359.

22. Shin CH, et al. Multiple roles for Med12 in vertebrate endoderm development. Dev Biol. 2008;317(2):467-479.

23. Wu SY, de Borsetti NH, Bain EJ, Bulow CR, Gamse JT. Mediator subunit 12 coordinates intrinsic and extrinsic control of epithalamic development. Dev Biol. 2014;385(1):13-22.

24. Rocha PP, Scholze M, Bleiss W, Schrewe H. Med12 is essential for early mouse development and for canonical Wnt and Wnt/ PCP signaling. Development. 2010;137(16):2723-2731.

25. Rocha PP, Bleiss W, Schrewe H. Mosaic expression of Med12 in female mice leads to exencephaly, spina bifida, and craniorachischisis. Birth Defects Res Part A Clin Mol Teratol. 2010;88(8):626-632.

26. Agah R, Frenkel PA, French BA, Michael LH, Overbeek PA, Schneider MD. Gene recombination in postmitotic cells. Targeted expression of Cre recombinase provokes cardiac-restricted, site-specific rearrangement in adult ventricular muscle in vivo. J Clin Invest. 1997;100(1):169-179.

27. Japp AG, Gulati A, Cook SA, Cowie MR, Prasad SK. The Diagnosis and Evaluation of Dilated Cardiomyopathy. J Am Coll Cardiol. 2016;67(25):2996-3010.

28. Xiao HB, Roy C, Fujimoto S, Gibson DG. Natural history of abnormal conduction and its relation to prognosis in patients with dilated cardiomyopathy. Int J Cardiol. 1996;53(2):163-170.

29. Krämer A, Green J, Pollard J, Tugendreich S. Causal analysis approaches in Ingenuity Pathway Analysis. Bioinformatics. 2014;30(4):523-530.

30. Mellström B, Savignac M, Gomez-Villafuertes R, Naranjo JR. Ca2+-operated transcriptional networks: molecular mechanisms and in vivo models. Physiol Rev. 2008;88(2):421-449.

31. Poss ZC, Ebmeier CC, Taatjes DJ. The Mediator complex and transcription regulation. Crit Rev Biochem Mol Biol. 2013;48(6):575-608

32. Ovcharenko I, Nobrega MA. Identifying synonymous regulatory elements in vertebrate genomes. Nucleic Acids Res. 2005;33(Web Server issue):W403-W407.

33. He A, Kong SW, Ma Q, Pu WT. Co-occupancy by multiple cardiac transcription factors identifies transcriptional enhancers active in heart. Proc Natl Acad Sci U S A. 2011;108(14):5632-5637.

34. Medina-Rivera A, et al. RSAT 2015: Regulatory Sequence Analysis Tools. Nucleic Acids Res. 2015;43(W1):W50-W56.

35. Lu J, McKinsey TA, Nicol RL, Olson EN. Signal-dependent activation of the MEF2 transcription factor by dissociation from histone deacetylases. Proc Natl Acad Sci U S A. 2000;97(8):4070-4075.

36. Pacini L, et al. Altered calcium regulation in isolated cardiomyocytes from Egr-1 knock-out mice. Can J Physiol Pharmacol. 
2013;91(12):1135-1142.

37. Chu G, et al. Enhanced myocyte contractility and $\mathrm{Ca} 2+$ handling in a calcineurin transgenic model of heart failure. Cardiovasc Res. 2002;54(1):105-116.

38. McKinsey TA, Zhang CL, Olson EN. MEF2: a calcium-dependent regulator of cell division, differentiation and death. Trends Biochem Sci. 2002;27(1):40-47.

39. Haberland M, Montgomery RL, Olson EN. The many roles of histone deacetylases in development and physiology: implications for disease and therapy. Nat Rev Genet. 2009;10(1):32-42.

40. Potthoff MJ, Olson EN. MEF2: a central regulator of diverse developmental programs. Development. 2007;134(23):4131-4140.

41. Carrera I, Janody F, Leeds N, Duveau F, Treisman JE. Pygopus activates Wingless target gene transcription through the mediator complex subunits Med12 and Med13. Proc Natl Acad Sci U S A. 2008;105(18):6644-6649.

42. Zhou H, Kim S, Ishii S, Boyer TG. Mediator modulates Gli3-dependent Sonic hedgehog signaling. Mol Cell Biol. 2006;26(23):8667-8682.

43. Sandmann T, et al. A temporal map of transcription factor activity: mef2 directly regulates target genes at all stages of muscle development. Dev Cell. 2006;10(6):797-807.

44. Grueter CE. Mediator complex dependent regulation of cardiac development and disease. Genomics Proteomics Bioinformatics. 2013;11(3):151-157.

45. Schiano C, Casamassimi A, Vietri MT, Rienzo M, Napoli C. The roles of mediator complex in cardiovascular diseases. Biochim Biophys Acta. 2014;1839(6):444-451.

46. Krebs $\mathrm{P}$, et al. Lethal mitochondrial cardiomyopathy in a hypomorphic Med30 mouse mutant is ameliorated by ketogenic diet. Proc Natl Acad Sci U S A. 2011;108(49):19678-19682.

47. Ito M, Yuan CX, Okano HJ, Darnell RB, Roeder RG. Involvement of the TRAP220 component of the TRAP/SMCC coactivator complex in embryonic development and thyroid hormone action. Mol Cell. 2000;5(4):683-693.

48. Landles $\mathrm{C}$, et al. The thyroid hormone receptor-associated protein TRAP220 is required at distinct embryonic stages in placental, cardiac, and hepatic development. Mol Endocrinol. 2003;17(12):2418-2435.

49. Jia Y, et al. Cardiomyocyte-Specific Ablation of Med1 Subunit of the Mediator Complex Causes Lethal Dilated Cardiomyopathy in Mice. PLoS One. 2016;11(8):e0160755.

50. Spitler KM, Ponce JM, Oudit GY, Hall DD, Grueter CE. Cardiac Med1 deletion promotes early lethality, cardiac remodeling, and transcriptional reprogramming. Am J Physiol Heart Circ Physiol. 2017;312(4):H768-H780.

51. Kobrynski LJ, Sullivan KE. Velocardiofacial syndrome, DiGeorge syndrome: the chromosome 22q11.2 deletion syndromes. Lancet. 2007;370(9596):1443-1452.

52. Muncke N, et al. Missense mutations and gene interruption in PROSIT240, a novel TRAP240-like gene, in patients with congenital heart defect (transposition of the great arteries). Circulation. 2003;108(23):2843-2850.

53. Clark RD, et al. FG syndrome, an X-linked multiple congenital anomaly syndrome: the clinical phenotype and an algorithm for diagnostic testing. Genet Med. 2009;11(11):769-775.

54. Graham JM, et al. Behavior of 10 patients with FG syndrome (Opitz-Kaveggia syndrome) and the p.R961W mutation in the MED12 gene. Am J Med Genet A. 2008;146A(23):3011-3017.

55. Mäkinen N, et al. MED12, the mediator complex subunit 12 gene, is mutated at high frequency in uterine leiomyomas. Science 2011;334(6053):252-255.

56. Lim WK, et al. Exome sequencing identifies highly recurrent MED12 somatic mutations in breast fibroadenoma. Nat Genet. 2014;46(8):877-880.

57. Rau MJ, Fischer S, Neumann CJ. Zebrafish Trap230/Med12 is required as a coactivator for Sox9-dependent neural crest, cartilage and ear development. Dev Biol. 2006;296(1):83-93.

58. Kim S, Xu X, Hecht A, Boyer TG. Mediator is a transducer of Wnt/beta-catenin signaling. J Biol Chem. 2006;281(20):14066-14075.

59. Tutter AV, et al. Role for Med12 in regulation of Nanog and Nanog target genes. J Biol Chem. 2009;284(6):3709-3718

60. Kagey MH, et al. Mediator and cohesin connect gene expression and chromatin architecture. Nature. 2010;467(7314):430-435

61. Aranda-Orgilles B, et al. MED12 Regulates HSC-Specific Enhancers Independently of Mediator Kinase Activity to Control Hematopoiesis. Cell Stem Cell. 2016;19(6):784-799.

62. Zhang H, Emmons SW. A C. elegans mediator protein confers regulatory selectivity on lineage-specific expression of a transcription factor gene. Genes Dev. 2000;14(17):2161-2172.

63. Yoda A, Kouike H, Okano H, Sawa H. Components of the transcriptional Mediator complex are required for asymmetric cell division in C. elegans. Development. 2005;132(8):1885-1893.

64. Amoasii L, et al. A MED13-dependent skeletal muscle gene program controls systemic glucose homeostasis and hepatic metabolism. Genes Dev. 2016;30(4):434-446.

65. Yin JW, et al. Mediator MED23 plays opposing roles in directing smooth muscle cell and adipocyte differentiation. Genes Dev. 2012;26(19):2192-2205.

66. Patel RK, Jain M. NGS QC Toolkit: a toolkit for quality control of next generation sequencing data. PLoS One. 2012;7(2):e30619.

67. Kim D, Pertea G, Trapnell C, Pimentel H, Kelley R, Salzberg SL. TopHat2: accurate alignment of transcriptomes in the presence of insertions, deletions and gene fusions. Genome Biol. 2013;14(4):R36.

68. Love MI, Huber W, Anders S. Moderated estimation of fold change and dispersion for RNA-Seq data with DESeq2. Genome Biol. 2014;15(12):550.

69. Liao Y, Smyth GK, Shi W. featureCounts: an efficient general purpose program for assigning sequence reads to genomic features. Bioinformatics. 2014;30(7):923-930.

70. Huang DW, et al. DAVID Bioinformatics Resources: expanded annotation database and novel algorithms to better extract biology from large gene lists. Nucleic Acids Res. 2007;35(Web Server issue):W169-W175

71. Nelson BR, et al. A peptide encoded by a transcript annotated as long noncoding RNA enhances SERCA activity in muscle. Science. 2016;351(6270):271-275. 
72. Zeng PY, Vakoc CR, Chen ZC, Blobel GA, Berger SL. In vivo dual cross-linking for identification of indirect DNA-associated proteins by chromatin immunoprecipitation. BioTechniques. 2006;41(6):694.

73. Zhao B, et al. The NF-kB genomic landscape in lymphoblastoid B cells. Cell Rep. 2014;8(5):1595-1606.

74. Langmead B. Aligning short sequencing reads with Bowtie. Curr Protoc Bioinformatics. 2010; Chapter 11:Unit 11.7.

75. Zhang Y, et al. Model-based analysis of ChIP-Seq (MACS). Genome Biol. 2008;9(9):R137.

76. Huber W, et al. Orchestrating high-throughput genomic analysis with Bioconductor. Nat Methods. 2015;12(2):115-121.

77. Robinson JT, et al. Integrative genomics viewer. Nat Biotechnol. 2011;29(1):24-26.

78. Black BL, Ligon KL, Zhang Y, Olson EN. Cooperative transcriptional activation by the neurogenic basic helix-loop-helix protein MASH1 and members of the myocyte enhancer factor-2 (MEF2) family. J Biol Chem. 1996;271(43):26659-26663.

79. Claycomb WC, et al. HL-1 cells: a cardiac muscle cell line that contracts and retains phenotypic characteristics of the adult cardiomyocyte. Proc Natl Acad Sci U S A. 1998;95(6):2979-2984. 